\title{
Sensory Modification of Leech Swimming: Rhythmic Activity of Ventral Stretch Receptors Can Change Intersegmental Phase Relationships
}

\author{
Jianhua Cang and W. Otto Friesen \\ Department of Biology, National Science Foundation Center for Biological Timing, University of Virginia, Charlottesville, \\ Virginia 22903-2477
}

\begin{abstract}
For segmented animals to generate optimal locomotory movements, appropriate phase relationships between segmental oscillators are crucial. Using swimming leeches, we have investigated the role of sensory input in establishing such relationships. We found that the stretch receptors associated with ventral longitudinal muscles encode the information of muscle contraction during swimming via membrane potential oscillations, with amplitudes of up to $10 \mathrm{mV}$ at our recording site. We subsequently modified the activity of ventral stretch receptors (VSRs) by injecting rhythmic current at different phases of the swim cycle and determined intersegmental phase lags by comparing the delay between the discharges of serially homologous motoneurons in three adjacent segments of isolated nerve cords. When no current was injected, the phase lag between neighboring segments
\end{abstract}

was $8.6 \pm 0.8^{\circ}$ (mean $\pm \mathrm{SEM} ; n=20$ ), with large phase variations from cycle to cycle, between different episodes, and between different preparations. When the phase of stretch receptor activity was set to $90-150^{\circ}$ by current injection, the phase of the motoneuron activity in the ganglion was consistently retarded by $\sim 5^{\circ}$. It was advanced by $\sim 5^{\circ}$ when the VSR phase was set to $240-300^{\circ}$. Therefore, the rhythmic activity of the ventral stretch receptor generated during swimming can change intersegmental phase lags of leech ganglia in a phase-dependent manner. These stretch receptors may set the optimal intersegmental phases during swimming movement in intact leeches.

Key words: Hirudo; locomotion; CPG; intersegmental coordination; motor control, sensory feedback
Rhythmic movements of animals are controlled by neural oscillators that provide the correct timing of motoneuron discharges. In nearly all systems studied thus far, the rhythmic patterns can be observed in the absence of sensory feedback (Delcomyn, 1980; Marder and Calabrese, 1996); that is, the neuronal network producing the rhythmic pattern, the central pattern generator (CPG), is located within the CNS. To achieve optimal mechanics during locomotion, however, the prototypical pattern generated by the CPG must be modified by sensory inputs that vary with individual body characteristics, development, and changes in the environment (Pearson, 1993; Pearson and Ramirez, 1997).

In the segmented animals, such as the leech, crayfish, and lamprey, central oscillators are found in most or all body segments (Cohen and Wallen, 1980; Weeks, 1981; Murchison et al., 1993; C. G. Hocker, X. Yu, and W. O. Friesen, unpublished observations). Leeches and other elongated animals, like lampreys and tadpoles, when swimming maintain a single-wavelength body form to achieve optimal swimming efficiency and stability (Gray, 1958; Kristan et al., 1974). To express the single wave requires appropriately phase-delayed muscle contractions in consecutive body segments. These contractions arise sequentially, because of phase lags between the activities of the segmental oscillators.

Experimental observations suggest that sensory input from the body wall increases the intersegmental phase lags generated by the CNS. When fictive motor patterns are generated in leech nerve cords isolated from sensory feedback, they display smaller intersegmental phase lags than those in intact animals (Kristan and Calabrese, 1976; Pearce and Friesen, 1984). Alternatively, in leeches with severed nerve cords, where sensory feedback alone

\footnotetext{
Received June 5, 2000; revised Aug. 7, 2000; accepted Aug. 9, 2000.

This research was supported by National Science Foundation Grant 97-23320 (W.O.F.). We thank Drs. Craig Hocker and Martin Straume for their assistance in data analysis and Karen Dame for expert editorial assistance. We also thank our colleagues Drs. Xintian Yu and Gisele Oda for their discussions.

Correspondence should be sent to W. Otto Friesen, Department of Biology, University of Virginia, Charlottesville, VA 22903-2477. E-mail: wof@virginia.edu. Copyright (C) 2000 Society for Neuroscience $0270-6474 / 00 / 207822-08 \$ 15.00 / 0$
}

generates intersegmental coordination, the intersegmental phase lags of the neuronal activity are larger than those of intact animals (Yu et al., 1999). The mechanism for interplay between sensory input and central oscillation is unknown.

Stretch receptors innervating the longitudinal muscle in each body segment [as described by Blackshaw (1993)] may modify the activity of the central swim oscillator. Three pairs of ventral stretch receptors (VSRs) are known to innervate ventral longitudinal muscles. These receptors hyperpolarize in response to stretch of the leech body wall (Blackshaw and Thompson, 1988). Hyperpolarization of the VSRs, in turn, changes the activity of swim-related motoneurons (Blackshaw and Kristan, 1990). Furthermore, recent studies have demonstrated that the VSRs interact with oscillatory interneurons (Cang et al., 1999). Thus, the VSRs are good candidates for providing phasic sensory input to modify intersegmental phase relationships.

To investigate the role of VSRs in influencing the intersegmental phase relationships of swimming activity, we first controlled the membrane potential of VSRs in isolated nerve cords by injecting rhythmic currents at different phases of the swim cycle; then we determined the phase relationships between segmental oscillators by comparing the delays between the discharges of serially homologous motoneurons. Statistical analyses of our electrical recordings demonstrate that the VSR activity does indeed change the local intersegmental phase lag. The results reveal a new function for sensory feedback in the modification of central pattern generation.

\section{MATERIALS AND METHODS}

Animals. Experiments were performed on adult medicinal leeches, Hirudo medicinalis, obtained from Biopharm (Charleston, NC). The leeches were maintained in artificial pond water in a controlled room on a $12 \mathrm{hr}$ light/dark cycle at $18-20^{\circ} \mathrm{C}$. During dissections, animals were anesthetized with cold leech saline $\left(4^{\circ} \mathrm{C}\right.$ ) containing (in $\mathrm{mM}$ ): $115 \mathrm{NaCl}, 4 \mathrm{KCl}, 1.8$ $\mathrm{CaCl}_{2}, 2 \mathrm{MgCl}_{2}, 10 \mathrm{HEPES}$ buffer.

The CNS of leech consists of the ventral nerve cord, which includes supraesophageal and subesophageal ganglia (the head ganglia), a chain of 21 metameric midbody ganglia, labeled M1 through M21, and a large posterior tail ganglion (denoted by "T"). Each midbody ganglion of the leech nerve cord contains cell bodies of bilaterally symmetrical neurons; 
we use "L" and " $\mathrm{R}$ " to indicate the left and right side of the ganglion, respectively. For example, $\mathrm{DP}(\mathrm{L}, 9)$ denotes the dorsal posterior nerve from the left side of midbody ganglion 9 (M9). Similarly, VSR(R,10) denotes the ventral stretch receptor on the right side of M10 (see Fig. $1 A$ ).

Physiology. Two types of preparations, isolated nerve cords and nerve cord-body wall preparations, were used (see Fig. 2). Both preparations consisted of the nerve cord from midbody ganglion 2 through the tail ganglion (M2-T). For nerve cord-body wall preparations, we removed a flap of the ventral body wall extending three segments, where only the middle segment was innervated by the nerve roots of one midbody ganglion; the two end segments were denervated. The body-wall flap was cut along the lateral midline and ventral midline so that only the VSRs on one side of the body were included (see diagram in Figs. $1 A, 2 A$ ). Dissected preparations were pinned out in a glass-bottom dish and perfused with leech saline, and the VSR axons, motoneurons, and interneurons were visualized with dark-field illumination. Both ends of the muscle flap were pinned down to the dish. The elastic property of the denervated muscle allows the muscle in the middle to contract even with the ends of the flaps immobilized. In these preparations, swim episodes were evoked by extracellular stimulation of dorsal-posterior nerves from a posterior ganglion, usually DP(16). During fictive swim episodes, the swim-related motoneurons in M2 through M18 undergo large oscillations in membrane potential. Impulse bursts of motoneurons were detected with extracellular suction electrodes placed on peripheral nerves. The most useful recording site, the DP nerve, exhibits bursts from the dorsal excitatory motoneuron, cell DE-3 (see Fig. 2). The median impulse of DP nerve bursts provides a convenient phase reference point for swim oscillations in each segment and is a reliable indicator of oscillator period and phase relationships (Kristan and Calabrese, 1976; Friesen, 1989).

Although there are three pairs of VSRs in each segment, we studied only one pair, those whose giant axons run near the anterior margin of the anterior root (Blackshaw and Thompson, 1988). We obtained intracellular recordings from this nonspiking axon with sharp electrodes (filled with $2 \mathrm{M}$ potassium acetate; resistance $40-50 \mathrm{M} \Omega$ ) while simultaneously recording DP nerve activity. An Axoclamp2A amplifier (Axon Instruments) was used to amplify the signals and inject currents.

In experiments to study whether the rhythmicity of a VSR can change intersegmental relationships, we obtained intracellular recordings from the VSR axon of a midbody ganglion, denoted as $n$ (between M8 and M12), and recorded DP nerve activity from that ganglion, the adjacent anterior ganglion $(n-1)$, and the adjacent posterior ganglion $(n+1)$ (see Fig. $3 A)$. With this set of electrodes in place, we manipulated the amplitude and phase of the VSR membrane potential in ganglion $n$ while recording swim bursts in the three ganglia. In addition, we obtained intracellular membrane potential recordings of motoneurons for use as a phase reference signal to control the timing of currents injected into the VSR. The oscillatory potential from motoneurons was led into a wave generator (Wavetek, San Diego, CA) to trigger the output of a single-cycle sinusoida wave on a cycle-by-cycle basis. The generator was set to produce a wave with a $2 \mathrm{nA}$ peak-to-trough amplitude. This sine wave was then injected into the VSR (see Fig. 3B). The period of the injected sinusoidal current was adjusted to approximate the intrinsic period of the fictive swimming in the nerve cord. By changing the trigger level of the wave generator, we were able to set the phase of the VSR activity over the full range of $360^{\circ}$ with respect to the swim cycle. As in our previous studies (Pearce and Friesen, 1984; Yu et al., 1999), the reference phase point $\left(0^{\circ}\right)$ for each swim cycle was assigned to the median impulse of each burst of DP nerves (indicated by $x$ in Fig. 3C). The cycle period was determined from the average time interval between the median impulses of consecutive swim bursts. Intersegmental phase lags (in degrees) between any two ganglia were calculated by dividing the time delay between bursts by the cycle period and then multiplying by $360^{\circ}$. To establish the phase of the VSR activity, we set the peaks of the VSR membrane potential as phase reference points (top trace of Fig. $3 C$ ) and compared these with the midpoints of DP bursts.

Ten to 36 swimming episodes were obtained from each preparation in which there either was no current injected into the VSR (control) or the currents were injected at various phases. Intersegmental phase lags were calculated for all swimming episodes.

Data analysis. Electrical signals recorded from the nerve cord were amplified and stored on magnetic tape for later analysis. Records on the tapes were digitized using a 12-bit analog-to-digital converter and analyzed by our customized software, Rhythm Analyses System (RAS, programmed by Dr. Craig Hocker, University of Virginia, Charlottesville, VA) in the environment of Matlab (MathWorks, Inc., Natick, MA) using circular statistics (Fisher, 1995)

For DP nerve records, individual swim bursts were identified by an RAS routine. Because each swim episode had 8-11 cycles, the value of calculated phase lag for each episode is associated with a SE $\left(\varphi_{i} \pm \sigma_{\varphi i}\right.$; see Table 1$)$.

To combine the data from different episodes and different animals, we used the variance-weighting method (Bevington, 1969). According to this method, the variance-weighted mean value, $\langle z\rangle$, of a collection of $n$ observables, $z_{i}$, each with corresponding uncertainty estimate (i.e., SD) $\sigma_{i}$ is given by the average of all $z_{i}$ weighted by the inverse of associated variance:

$$
\langle z\rangle=\left(\sum_{i=1}^{n}\left(z_{\mathrm{i}} / \sigma_{i}^{2}\right)\right) /\left(\sum_{i=1}^{n}\left(1 / \sigma_{i}^{2}\right)\right) .
$$

To obtain the corresponding variance-weighted SE of the varianceweighted mean, the deviation of individual $z_{i}$ from $\langle z\rangle,\left(z_{i}-\langle z\rangle\right)^{2}$, is also weighted by the inverse of associated variance:

$$
\sigma_{\langle z\rangle}=\left[\sum_{i=1}^{n}\left(\left(z_{i}-\langle z\rangle\right)^{2} / \sigma_{i}^{2}\right) /(n-1) \sum_{i=1}^{n}\left(1 / \sigma_{i}^{2}\right)\right]^{1 / 2} .
$$

Because the phase lag of each episode is associated with a $\operatorname{SE}\left(\varphi_{i} \pm \sigma_{\varphi i}\right.$; see Table 1), it is appropriate to use the variance-weighting method. The control value of the intersegmental phase lag of each animal, $\langle c\rangle \pm \sigma_{<\mathrm{c}>}$, is the variance-weighted mean of the phase lags of the control episodes (see Table 1). To combine the results from different leeches, the data were normalized by subtracting the control values of individual animals $\left(\varphi_{\mathrm{ni}}=\right.$ $\varphi_{\mathrm{i}}-\langle c>)$. The $\mathrm{SE}$ of each normalized data point is given as $\sigma_{n i}=\left(\sigma_{\varphi \mathrm{i}}{ }^{2}\right.$ $\left.+\sigma_{<c>}^{2}\right)^{1 / 2}$

\section{RESULTS}

\section{Identification of the ventral stretch receptors}

As the first step in a series of investigations of how sensory input from the body wall modifies swimming rhythm, we identified and recorded intracellularly from a pair of VSRs with giant axons lying near the anterior margin of the anterior root (Fig. 1). The axons, previously characterized by Blackshaw and Thompson (1988), cannot sustain action potentials, although their cell bodies can. Hyperpolarization of the receptor soma induced by stretching the body wall transmits electrotonically to nerve terminals within the ganglion.

Dual penetrations were made of the identified pair of VSRs in M10 (Fig. 1A). On the right side, a flap of ventral body wall was included in the preparation to keep the soma and dendrites of $\operatorname{VSR}(\mathrm{R}, 10)$ intact. The small spikes characteristic of the VSRs (Blackshaw and Thompson, 1988) can be seen in both left- and right-side records, with similar amplitudes and frequencies. We verified that the VSRs hyperpolarize in response to stretch of ventral body wall (X. Yu, personal communication). Furthermore, intracellular dye injection (data not shown) revealed the terminal arborizations of the VSR within the segmental ganglion, consistent with those described by Blackshaw and Thompson (1988). We conclude that the giant axons at the anterior margin of the anterior nerve root can be identified as VSR axons by their position and impulse characteristics even when the soma and dendrites are removed.

We observe that the small spikes recorded from the VSRs in the isolated nerve cord (Figs. $1 B, 2 B$ ) must originate from its terminals within the ganglion, based on the absence of soma in this preparation and the characteristic inability of the axon to sustain an action potential. The frequency of these small spikes varied between preparations, depending on the membrane potential, and was highly sensitive to current injection. The response to current injection is shown in the example in Figure $1 B$, where hyperpolarizing current $(0.1 \mathrm{nA})$ injected into the VSR abolished the small spikes, and small depolarizing currents increased their frequency, indicating that these spontaneous potentials are most likely attenuated action potentials initiated near the central terminals of the VSRs (see Discussion).

\section{Activity of ventral stretch receptors during fictive swimming}

To participate in modifying the swimming rhythm, the VSRs would have to convey sensory information from the body wall. We examined the activity during fictive swimming to ascertain whether VSRs do transmit phasic information. In muscle-attached preparations, the membrane potential of VSRs oscillated during swimming, with amplitudes up to $10 \mathrm{mV}$ at our recording site, the axon near the ganglion. In the example shown in Figure $2 A$, the phase of the VSR membrane potential oscillation is $\sim 140^{\circ}$ compared with the DP nerve bursts from the same ganglion (using the most 
A

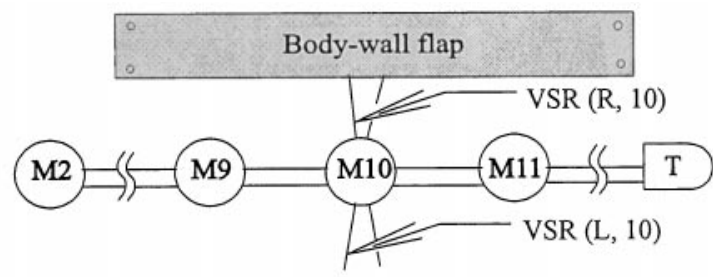

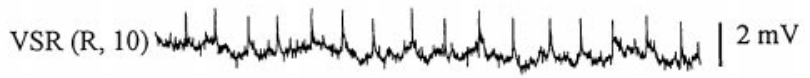

$\operatorname{VSR}(\mathrm{L}, 10)$

B

VSR $(L, 12)$ without muscle
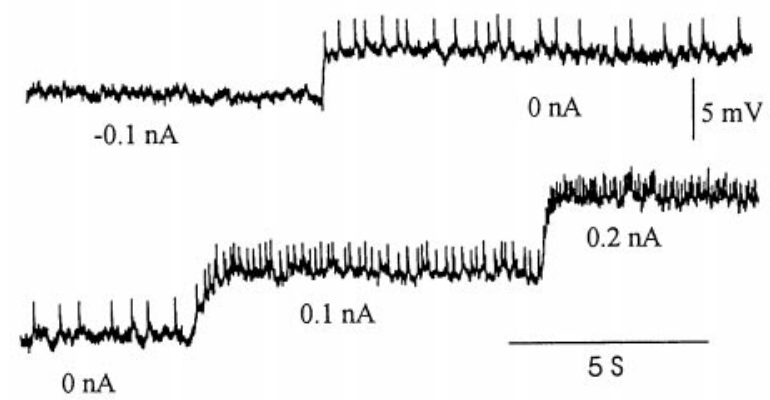

Figure 1. Intracellular recordings from VSRs. $A$, Intracellular recordings obtained from VSR axons in the nearly isolated nerve cord. The experimental setup is diagramed at the top: nerve cord from midbody ganglion 2 through the tail ganglion $(M 2-T)$. Dual penetrations were made from the pair of identified VSRs of M10. On the right side only, a flap of ventral body wall was included in the preparation to keep the soma and dendrites of $\operatorname{VSR}(R, 10)$ intact. With or without the intact muscle, the characteristic small spikes of the VSR can be seen, with similar amplitudes $(2 \mathrm{mV})$ and frequencies $(\sim 10 \mathrm{~Hz})$ in both records, indicating that the small spikes originate from VSR terminals within the ganglion and that it is not necessary to include innervated muscle to identify the VSR. $B$, Spike frequency of the VSR is sensitive to the current injection. Continuous intracellular records from a VSR of ganglion $12[\operatorname{VSR}(L, 12)]$ from another preparation with no attached muscle show that small injected currents changed the spike frequency dramatically.

depolarized point as the phase reference marker). The phase of the VSR varied with respect to the DP nerve bursts from 100 to $150^{\circ}$ for 12 different episodes in three preparations.

To determine whether the oscillations in VSR membrane potential observed during fictive swimming were indeed generated in the periphery, we recorded from VSRs in nerve cord preparations isolated from the muscle for comparison. Although most of the recordings from the isolated nerve cords did show VSR membrane potential oscillation, as in Figure $2 B$, the amplitude was smaller than that observed when the muscle was attached and the phase differed with respect to DP nerve bursts $\left(\sim 0^{\circ}\right)$ (Fig. 2, compare $A$ and $B$ ). Because the VSR oscillations displayed a greater amplitude and different phase when the VSR soma was in contact with muscle during swimming, we concluded that the oscillations shown in Figure $2 A$ were indeed caused by peripheral input, specifically by rhythmic muscle contraction. In addition, the fact that there were any VSR membrane potential oscillations observed, however small, in the completely isolated nerve cord during fictive swimming (Fig. $2 B$ ) is consistent with earlier observations that the VSRs receive synaptic inputs from swim-related oscillatory interneurons (Cang
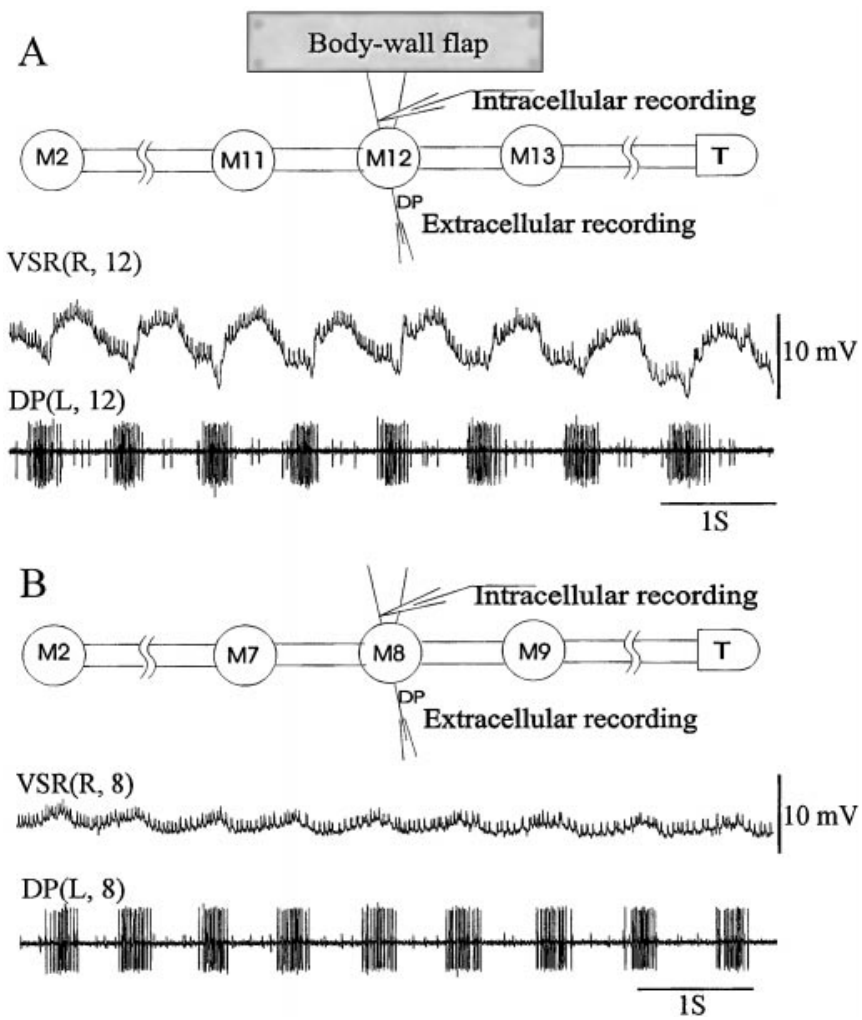

Figure 2. VSR activity during swimming. $A$, Membrane potential oscillation of a VSR in a CNS-muscle preparation (M2-T, the right ventral body wall of segment 12 was innervated by the segmental nerves, diagrammed at the top $)$. The first trace $[\operatorname{VSR}(R, 12)]$ is an intracellular record of a ventral stretch receptor. The second is an extracellular trace $[D P(L, 12)]$ obtained from the same ganglion to monitor swimming rhythm. The membrane potential of the VSR oscillated during swimming $(10 \mathrm{mV}$ amplitude, phase of $\sim 140^{\circ}$ ). Note that the frequency of small spikes varies with the membrane potential oscillation (but less than expected from the response of the specific example in Fig. $1 B$ ). $B$, Activity of a VSR during fictive swimming in the isolated nerve cord (diagrammed at the top). The membrane potential of the VSR (top trace) oscillates, but with smaller amplitude (1-2 mV) and different phase $\left[\sim 0^{\circ}\right.$ with respect to the $D P(L, 8)$ record] than when the soma and dendrites are intact.

et al., 1999). These central inputs may be able to control the gain of sensory information inflow.

\section{Modification of intersegmental phase lag by the ventral stretch receptor}

To investigate whether, during swimming, VSR activity influences the intersegmental phase relationship, we mimicked oscillatory VSR activity by injecting sinusoidal current into the VSR, as described in Materials and Methods and in Figure 3, and examined intersegmental phase lags.

In a control experiment, data from a fictive swimming episode were acquired in the manner shown in Figure $3 A$ but without any current injection. Figure $4 A$ shows that the instantaneous phase lag between the two ganglia $n$ and $n+1$ [abbreviated as phase lag ( $n$, $n+1)$ ] varies considerably throughout the episode. To reduce the complexity of data, we used the mean phase lag of each episode as one single datum point. Each swim episode had 8-11 cycles, so the calculated phase lag for each episode is associated with a SE $(\varphi \pm$ $\left.\sigma_{\varphi}\right)$ (Table 1$)$. Figure $4 B$ shows the complete set of phase lag ( $n$, $n+1)$ measurements from the same experiment, including several control episodes and the experimental episodes, in which the VSR phase with respect to the DP nerve bursts was manipulated. Phase lags (in degrees) of $(n, n+1)$ are plotted against the phase of VSR activity with respect to the DP nerve bursts. The phase lags for control episodes in this preparation were between 0.3 and $7.4^{\circ}$, with a mean of $4.4^{\circ}$. For the episodes in which currents were injected into the VSR, the phase lag $(n, n+1)$ ranged from -8.1 to $13.7^{\circ}$, 


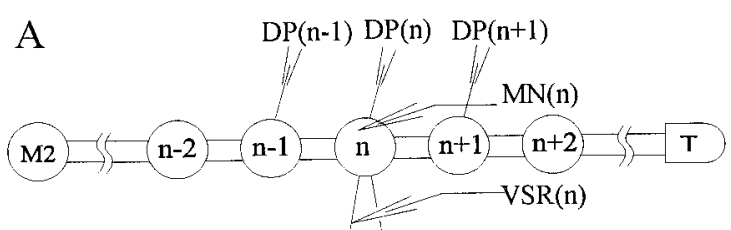

B
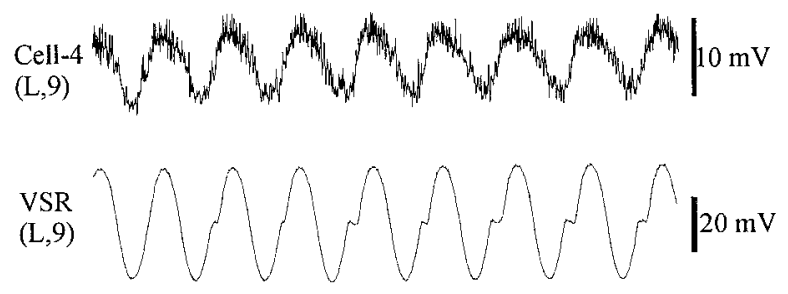

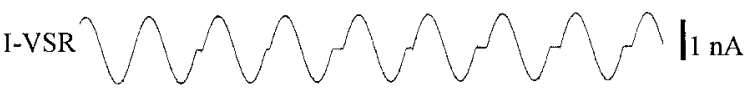
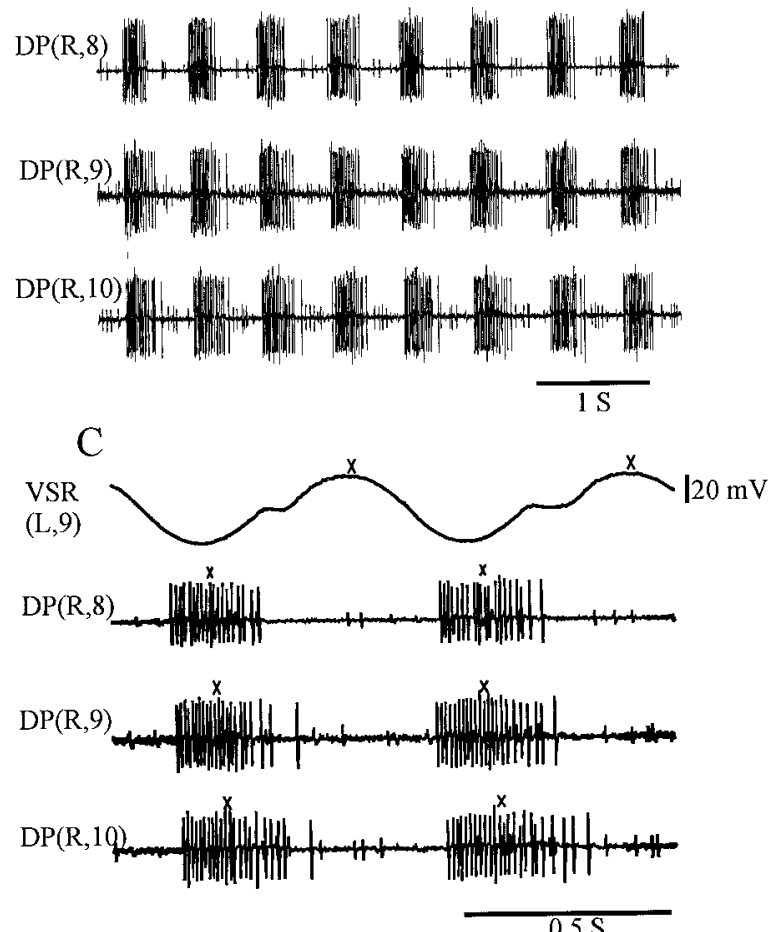

Figure 3. Modification of intersegmental phase relationships by VSR activity. $A$, Diagram of the experimental setup. $D P$, Dorsal posterior nerve; $M 2$, midbody ganglion 2; $T$, tail ganglion; $M N$, motoneuron; $V S R$, ventral stretch receptor; $n$ designates the stimulated ganglion, which was M8 in the example shown below. $B$, Sample records. Top trace shows the membrane potential of the motoneuron (Cell-4 in this example) used to trigger the wave generator during each swim cycle to inject a single cycle of sinusoidal current into the VSR. Second trace shows the intracellular recording from the VSR axon. Third trace shows current injected into the VSR. The period of the sine wave was set to approximate the intrinsic period of fictive swimming (current amplitude was $\sim 2 \mathrm{nA}$ peak-to-trough, without DC offset). Because the swim cycle periods were slightly longer than that of the injected current, short notches are present in the second and third traces Bottom three traces, Extracellular recordings from $D P(R, 8), D P(R, 9)$, and $D P(R, 10)$, respectively. $C$, Calculation of phase relationship. Expanded section of the traces in $B$, including $\operatorname{VSR}(L, 9)$ and three traces of $D P$ nerve records. Phase reference points ( $x$ 's) were assigned to the median impulse of each burst of the DP nerve, and the peak of VSR membrane potentialimpulses are not visible. The cycle period was determined from the average time interval between the median impulses of consecutive swim bursts. The phase lag (in degrees) was calculated by dividing the time delay between phase reference points by the cycle period and then multiplying it by $360^{\circ}$.

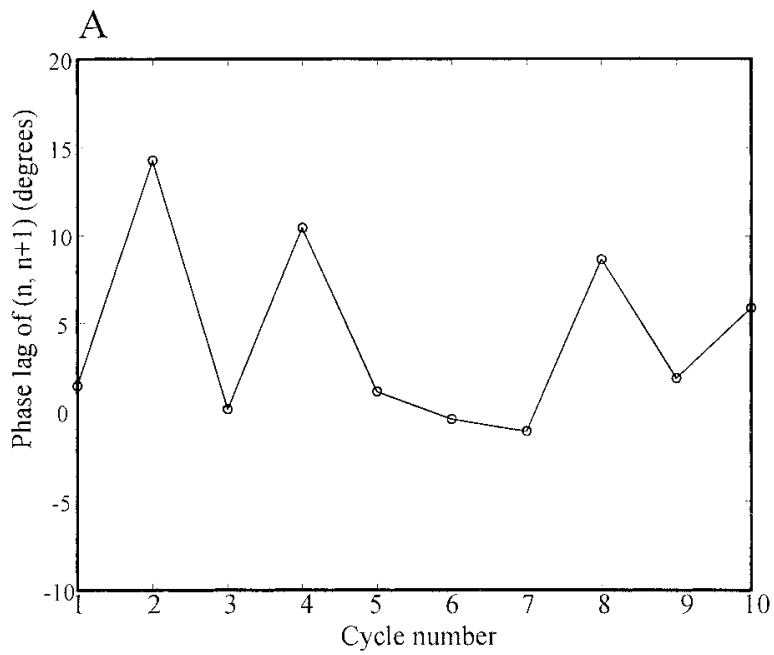

$\mathrm{B}$

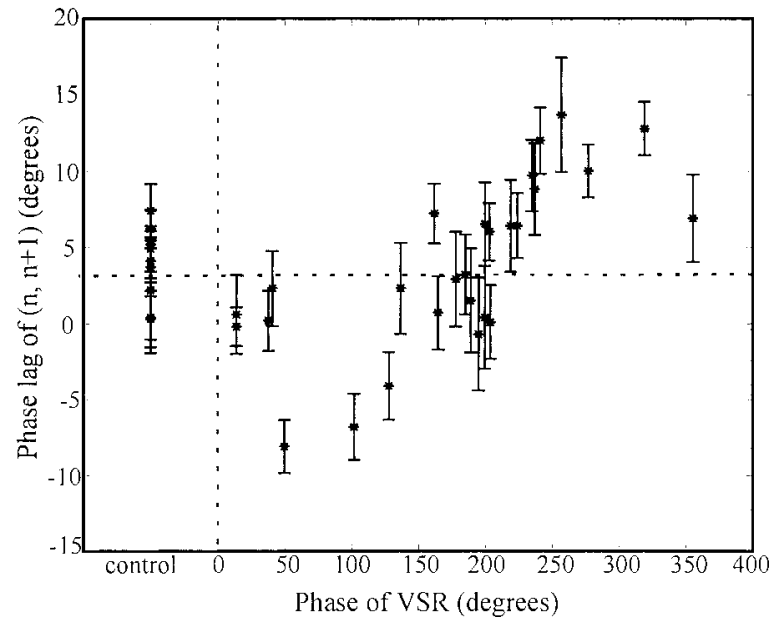

Figure 4. Example of phase lag between ganglion $n$ and $n+1$ in one isolated nerve cord preparation. We recorded from VSR(R,11), DP(L,11), and $\operatorname{DP}(\mathrm{R}, 12)$ under the conditions described in Figure 3 and calculated the phase lag between ganglia $n$ and $n+1$ [abbreviated as phase lag $(n, n+1)$, $n=\mathrm{M} 11$ in this case] for 36 swim episodes. $A$, Instantaneous phase plot of a control episode (i.e., no phase imposed on the VSR). The phase lag ( $n$, $n+1)$ is plotted against swim-cycle number. The intersegmental phase lag varies from 0 to $15^{\circ}$, with mean of $4.6^{\circ} . B$, Phase lag plot of the complete data set from the experiment. The phase lag $(n, n+1)$ of each episode is plotted against the phase of VSR activity. The error bar of each point represents the SE of each swimming episode (8-11 cycles). The phase lags of the nine control episodes ranged from 0.3 to $7.4^{\circ}$, with a mean value of $4.4^{\circ}$ (horizontal dotted line). The phase lag $(n, n+1)$ first decreased, as VSR phase was increased from 0 to $50^{\circ}$, then increased until VSR phase was $\sim 250^{\circ}$, and finally decreased to near control values as VSR phase further increased to $360^{\circ} / 0^{\circ}$.

varying in a phase-dependent manner as VSR phase was varied. More specifically, the phase lag $(n, n+1)$ increased as much as $10^{\circ}$ from control when the phase of the VSR membrane potential was in the range of $240-330^{\circ}$ and decreased $10^{\circ}$ when the phase of VSR was $\sim 90^{\circ}$. It is clear that in this single preparation the VSR activity is able to modulate the intersegmental phase lag between ganglion $n$ and $n+1$. To examine the observations more precisely, we repeated the experiments on 19 additional preparations.

\section{The intersegmental phase lag of isolated nerve cords in control experiments}

We first examined the intersegmental phase lag in the middle of leech nerve cord (M7-M13) when there was no current injected into the VSRs (control experiments). We had two measurements of the intersegmental phase lag from most preparations: phase lag $(n-1, n)$ and phase lag $(n, n+1)$. To study the intersegmental phase 


\begin{tabular}{|c|c|c|}
\hline & Mean & SE \\
\hline Phase lag of individual episodes & $\begin{array}{l}\varphi_{i} \text { for each episode, given by the } \\
\text { mean of all cycles of the episode }\end{array}$ & $\begin{array}{l}\sigma_{\varphi \mathrm{i}} \text {, transformed to SD for use in } \\
\text { Equations } 1 \text { and } 2\end{array}$ \\
\hline Control value of one leech & $\begin{array}{l}\langle c\rangle \text {, given by Equation } 1 \text { from all } \\
\text { control episodes of the leech }\end{array}$ & $\sigma_{\langle\mathrm{c}\rangle}$, given by Equation 2 \\
\hline Data after normalization & $\varphi_{\mathrm{ni}}=\varphi_{\mathrm{i}}-\langle\mathrm{c}\rangle$ for each episode & $\sigma_{\mathrm{ni}}=\left({\sigma_{\varphi \mathrm{i}}}^{2}+\sigma_{\langle\mathrm{c}\rangle}^{2}\right)^{1 / 2}$ \\
\hline
\end{tabular}

lag under the control condition, it is not necessary to differentiate between the phase lags $(n-1, n)$ and $(n, n+1)$, so both are included in our analyses.

From 20 different leeches we obtained values of the intersegmental phase lag $\left(\varphi_{\mathrm{i}}\right)$ from 273 control episodes. The distribution of all control values is plotted in Figure $5 A$. The phase lags of individual episodes distribute around $8^{\circ}$, ranging from -4 to $24^{\circ}$. We reduced these data by first calculating means $(<c>)$ and SEs $\left(\sigma_{<\mathrm{c}>}\right)$ for each of the 20 preparations using the variance-weighting method (see Materials and Methods). The mean phase lag of each preparation ranged from 3.4 to $20.7^{\circ}$, with a mean for all preparations of $8.6 \pm 0.8^{\circ}$ (variance-weighting mean $\pm \mathrm{SE}$ ). We then normalized the control data for each preparation by subtracting the mean phase lag from observed phase lag values. The distribution of the normalized control data $\left(\varphi_{\mathrm{ni}}=\varphi_{\mathrm{i}}-\langle c\rangle\right)$ is shown in Figure $5 B$. It can be seen that the phase lag of each episode is normally distributed around the mean phase lag $\left(0^{\circ}\right.$ for the control values shown in the Figure) and ranges from -11 to $9^{\circ}$. Next, we used normalized data to analyze whether VSR activity changed intersegmental phase lags.

\section{Rhythmic activity of the VSR can change intersegmental phase lag}

Phase lag between ganglia $\mathrm{n}$ and $\mathrm{n}+1$

We performed the VSR phase experiments as illustrated for a single preparation in Figure 4B on 20 different leeches and observed similar results from nearly every preparation. Figure $6 \mathrm{~A}$ shows the normalized phase lags $(n, n+1)$ of all episodes from these leeches plotted against the phase of VSR activity. In this Figure, the circles represent the normalized phase lag of control episodes, which distribute $\sim 0^{\circ}$. The combined data for experimental episodes (represented by $x$ 's) in Figure $6 A$ exhibit the same trend as the single preparation shown in Figure $4 B$. The phase lag $(n, n+1)$ decreased when the phase of the VSR activity was between 0 and $180^{\circ}$ and increased between 180 and $360^{\circ}$. The changes from the control value were as large as $10^{\circ}$ when the VSR phase was between 120 and $270^{\circ}$. Because a period change could slightly alter the phase lag (Pearce and Friesen, 1984), we also plotted the periods of the corresponding episodes (Fig. 6B). Clearly, all phases of VSR activity were tested equally over the small range of periods generated by these isolated nerve cord preparations. Also, these data demonstrate that cycle period in the isolated nerve cord is not influenced by rhythmic VSR activity ( $p>0.05$, using the method described below).

To examine the above observation statistically, we grouped the data for phase lag $(n, n+1)$ into bins, each representing $30^{\circ}$ intervals of phase for VSR activity, and performed a Kruskal-Wallis nonparametric ANOVA test and Dunn's multiple comparison post-test (for those data where the $p$ value of the ANOVA was $<0.05)$ (Fig. 7). The phase lags of the bins of $90-120^{\circ}\left(-4.9 \pm 1.6^{\circ}\right.$, mean \pm SEM $)$ and $120-150^{\circ}\left(-5.4 \pm 0.6^{\circ}\right)$ are significantly smaller than that of control (Kruskal-Wallis nonparametric ANOVA test: $p<0.0001$; Dunn's multiple comparison post test: ${ }^{*} p<0.05$ and $* * * p<0.001$, respectively, in Fig. 7), and those of the bins of $240-270^{\circ}\left(4.6 \pm 0.6^{\circ}\right)$ and $270-300^{\circ}\left(4.9 \pm 0.8^{\circ}\right)$ are significantly larger ( $p<0.001$ for both groups). Therefore, our data demonstrate that the activity of the VSR in ganglion $n$ can increase or decrease the intersegmental phase lag between ganglia $n$ and $n+1$, depending on the phase of the VSR.

\section{Evaluation of the phase lags $(n-1, n)$, and $(n-1, n+1)$}

The change of the phase lag $(n, n+1)$ by the VSR activity, as described above and in Figure 7, may result from the phase shift of the oscillator in ganglion $n$, ganglion $n+1$, or both. To determine which one is the case, we analyzed the phase lag between ganglia $n-1$ and $n$.

The outcomes were the opposite of those for the phase lag ( $n$, $n+1)$ in Figure 7. As shown in Figure $8 A$, the phase lag $(n-1, n)$ was significantly larger than the control value when the phase of the VSR activity was in the range of $90-120^{\circ}\left(2.6 \pm 1.3^{\circ}, p<0.05\right)$ and $120-150^{\circ}\left(3.7 \pm 0.9^{\circ}, p<0.01\right)$, and smaller than control in the range of $240-270^{\circ}\left(-3.7 \pm 0.7^{\circ}, p<0.001\right)$ and $270-300^{\circ}(-4.2 \pm$ $1.0^{\circ}, p<0.05$ ) (Kruskal-Wallis nonparametric ANOVA test: $p<$ $0.0001)$

Comparison of Figures 7 and $8 A$ illustrates that the phase-lag changes induced by the VSR stimulation differ between the pairs of ganglia $(n, n+1)$ and $(n-1, n)$, falling to similar degrees in the opposite direction and hence appearing to, in effect, cancel each other out. To test whether this is true, we plotted the change of phase lag $(n-1, n+1)$ against the phase of VSR activity (Fig. $8 B)$. In
Figure 5. Distribution of intersegmental phase lags of control episodes. In experimental setup as described in Figure 3, phase lags were measured in 6-12 control episodes for each of 20 preparations in which no current was injected into the VSRs. Two measurements of intersegmental phase lag, phase lag $(n-1, n)$ and phase lag $(n, n+1)$, were obtained for each episode from most preparations. $A$, Distribution of the phase lags of all control episodes ( 273 values from 20 preparations). The ordinate gives the number of occurrences for each phase lag observed. The phase lags of all individual episodes distribute around $8^{\circ}$, ranging from -4 to $24^{\circ} . B$, Distribution of the normalized data. The data of all control episodes for each preparation were normalized by subtracting the corresponding mean phase lag. The normalized phase lags of all episodes are normally distributed around the mean phase lag $\left(0^{\circ}\right.$ after normalization), with a range from -11 to $9^{\circ}$.

\section{A Original data}

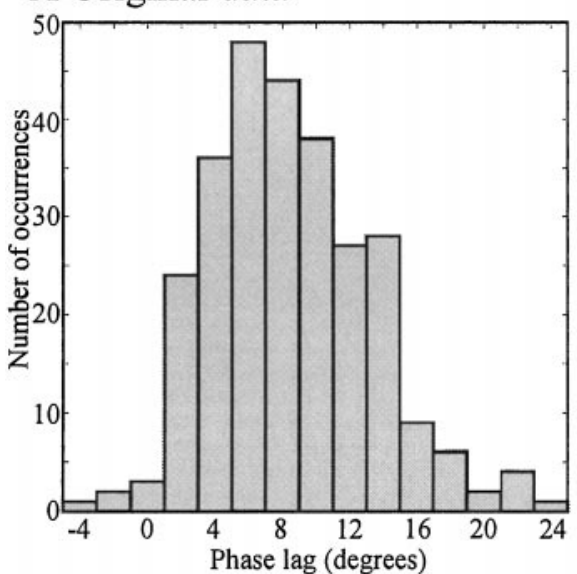

\section{B Normalized data}

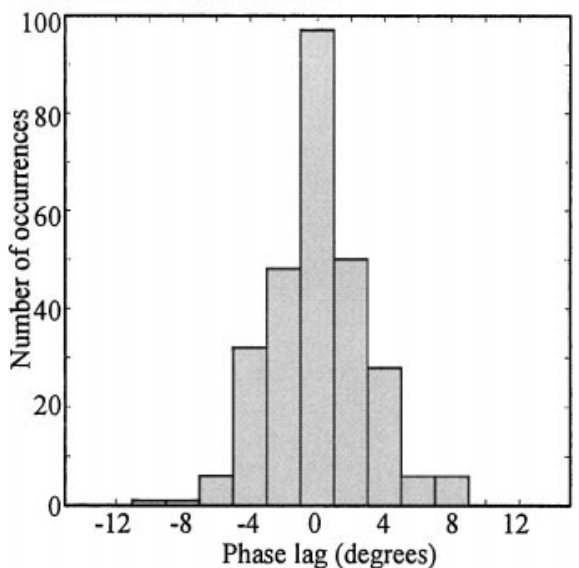




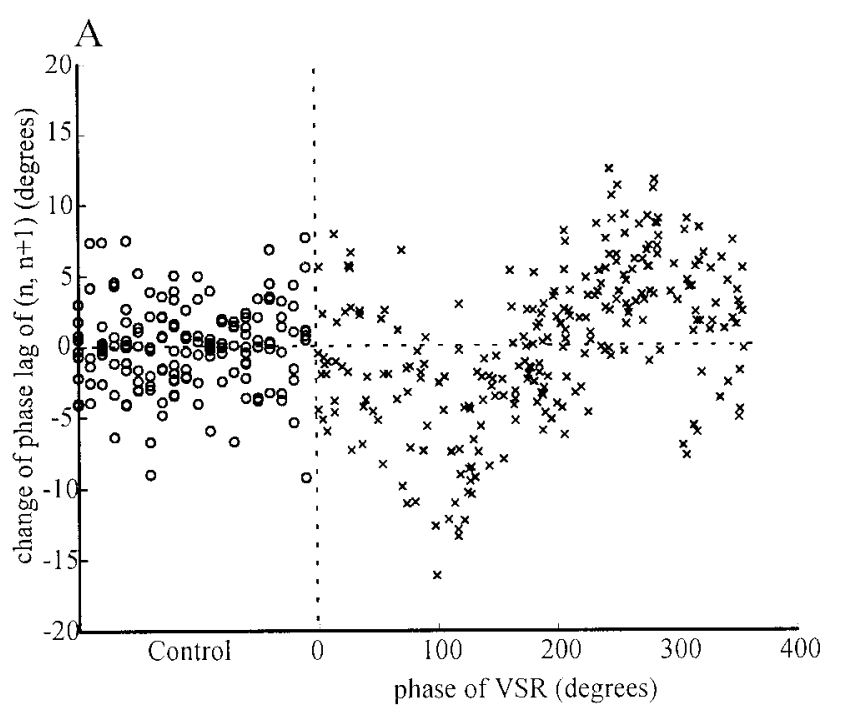

B

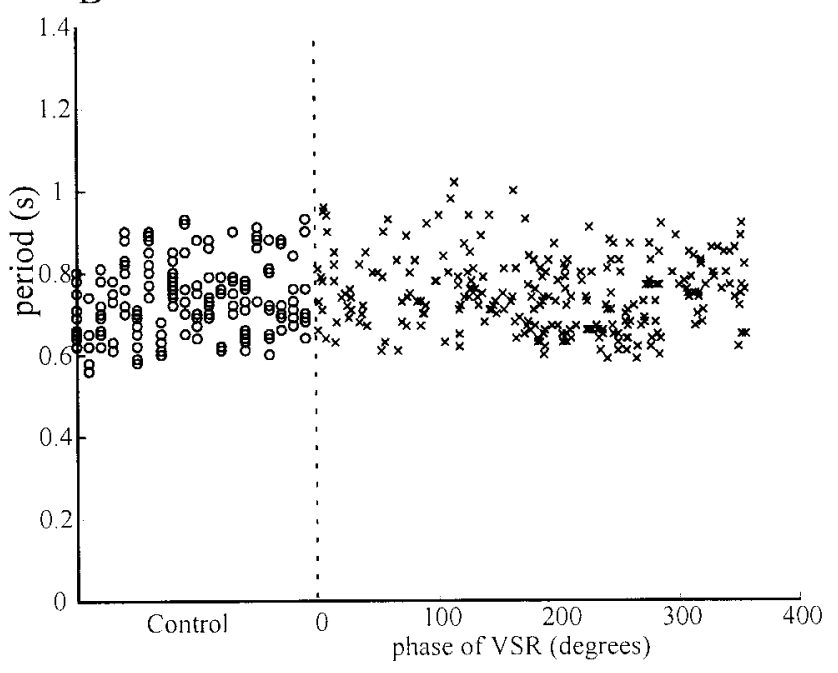

Figure 6. Normalized phase lags between ganglia $n$ and $n+1$. $A$, Normalized phase lags between ganglia $n$ and $n+1$. The original phase lag $(n, n+1)$ data for all episodes from 20 experiments conducted as described in Figure 3 were normalized by subtracting from each episode the mean phase lag of the corresponding preparation, and the results were plotted against the induced phase of VSR activity. The SE of each point, $\sigma_{n i}=\left(\sigma_{\varphi \mathrm{i}}{ }^{2}+\sigma_{<\mathrm{c}>}\right)^{1 / 2}$ was calculated but is not shown in the figure for clarity. Although there is considerable scatter in the data, the phase lag $(n, n+1)$ increased maximally when the VSR phase was near $270^{\circ}$ and decreased most when the phase was near $120^{\circ}$. The horizontal dotted line indicates the normalized mean phase of control episodes, $0^{\circ}$. $B$, Cycle period and VSR phase. The periods of all episodes are plotted against the VSR phase. The same range of swim-cycle periods occurred at each value of VSR phase. In both $A$ and $B, \bigcirc$ represents the data from control episodes, and $x$ represents those from experimental episodes. The control and experimental data are separated by vertical dotted lines.

the resulting graph, only small variations in the phase lag are apparent, and these do not differ significantly from phase-lag values in control experiments, in which the phasic activity of the VSR was absent $(p$ value $=0.067$ for the ANOVA).

Therefore, these results demonstrate that the changes in intersegmental phase lags resulting from the VSR response to stimulation are attributable mainly to the effects within the ganglion containing the stimulated VSR.

\section{DISCUSSION}

Previous studies of leech swimming focused on identifying swim oscillator circuits and their intersegmental connections within the CNS (Friesen, 1989; Brodfuehrer et al., 1995). However, the prototypical pattern generated by the leech swimming CPG is insuf-

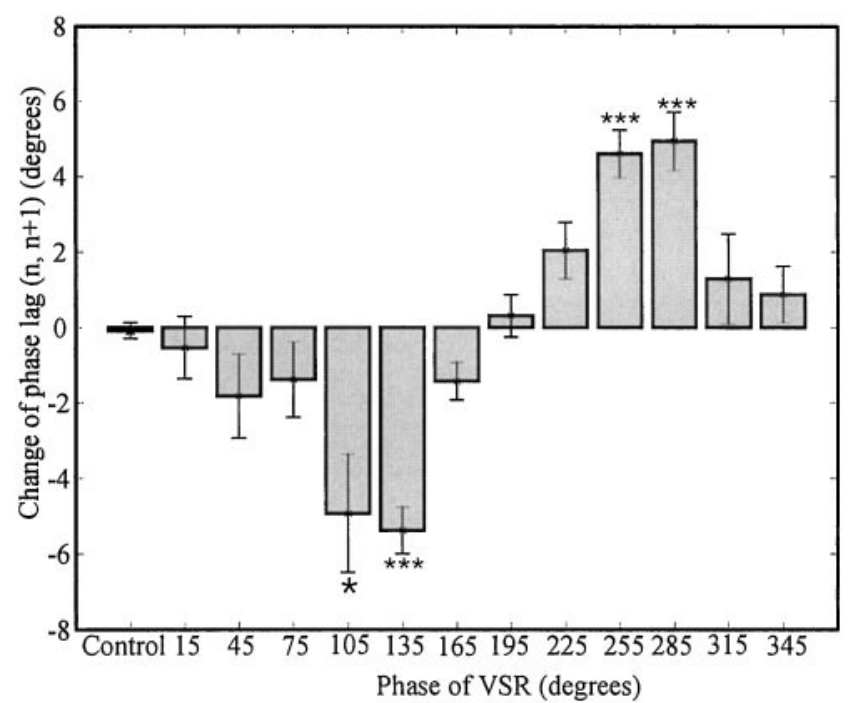

Figure 7. Phase lag changes between ganglia $n$ and $n+1$ caused by VSR activity. The data in Figure $6 A$ were grouped into 13 bins according to the phase of VSR. The first bin includes all the data from control episodes; the bin widths for experimental data are $30^{\circ}$. The mean and SE of each bin and control group were determined by the variance-weighting method. The phase lags of the bins of $90-120^{\circ}\left(-4.9^{\circ} \pm 1.6^{\circ}\right.$, mean \pm SEM $)$ and $120-150^{\circ}$ $\left(-5.4 \pm 0.6^{\circ}\right)$ are significantly smaller than that of control (Kruskal-Wallis nonparametric ANOVA test: $p<0.0001$; Dunn's multiple comparison post test: $* p<0.05$ and $* * * p<0.001$, respectively), and those of the bins of $240-270^{\circ}\left(4.6 \pm 0.6^{\circ}\right)$ and $270-300^{\circ}\left(4.9 \pm 0.8^{\circ}\right)$ are significantly larger $(p<$ 0.001 for both groups).

ficient for producing the correct movement pattern without sensory inputs because the intersegmental phase lag is too small to account for a one-wavelength waveform (Pearce and Friesen, 1984). In addition, the leech must adjust the intersegmental activity delay in response to environmental and developmental changes.

We have now begun to investigate the specific neuronal mechanisms for sensory control of intersegmental phase lags in the leech by studying the effects of ventral stretch receptor activity. Our experiments show that VSRs encode peripheral phase information during swimming via membrane potential oscillation and that the rhythmic activity of a VSR caused by current injection can change the local intersegmental phase lag by delaying or advancing the phase of segmental oscillator in a phase-dependent manner. During leech swimming, muscle contractions can be expected to generate similar rhythmic VSR activity and thereby alter intersegmental phase lags. Our investigation, therefore, has revealed a new role of sensory feedback in the modification of central pattern generation.

\section{The VSR is a viable candidate to mediate sensory input during swimming}

Most of the identified sensory neurons in the leech are unlikely candidates for the mediation of phasic sensory inputs to the $\mathrm{CPG}$. These include the touch, pressure, and nociceptive mechanosensory neurons, which are usually silent during swimming (Yu et al., 1999), and the sensillar movement receptors, which are anatomically and functionally not suitable for carrying phase information from the periphery (Friesen, 1981). The likely mediators of phasic sensory input are the stretch receptors associated with longitudinal muscles for the following reasons. First, the membrane potential of VSRs oscillates when the longitudinal muscle contracts rhythmically (Fig. 2A). Second, the VSRs make synaptic connections with swim oscillator interneurons (Cang et al., 1999). Finally, imposed rhythmic activity in the VSR modifies intersegmental phase relationships in a phase-dependent manner (Figs. 7, 8). The dorsal stretch receptors (Blackshaw, 1993) may also carry the phasic information of periphery and interact with interneurons, so they would also contribute to intersegmental coordination during swimming. 
Figure 8. Phase changes between ganglia $n-1$ and $n$, and between ganglia $n-1$ and $n+1$. Data were obtained by the same methods used for Figure 7. $A$, Bar graph of phase changes between ganglia $n-1$ and $n$. The mean phase lag of each group was plotted against the phase of VSRs. The phase lags of the bins of $90-120^{\circ}\left(2.6 \pm 1.3^{\circ}\right.$, mean \pm SEM) and $120-150^{\circ}\left(3.7 \pm 0.9^{\circ}\right)$ are significantly larger than that of control (ANOVA test: $p<0.0001$; Dunn's multiple comparison post test: ${ }^{*} p<0.05$ and ${ }^{* *} p<0.01$ respectively), and those of the bins of $240-270^{\circ}\left(-3.7 \pm 0.7^{\circ}\right)$ and $270-300^{\circ}\left(-4.2 \pm 1.0^{\circ}\right)$ are significantly smaller ( $p<0.001$ and $p<0.05$, respectively). $B$, Phase lags between ganglia $n-1$ and $n+1$. Although there are small variations, the activity of VSR did not change the phase lag between ganglia $n-1$ and $n+1$ significantly (ANOVA test: $p=0.067$ ).
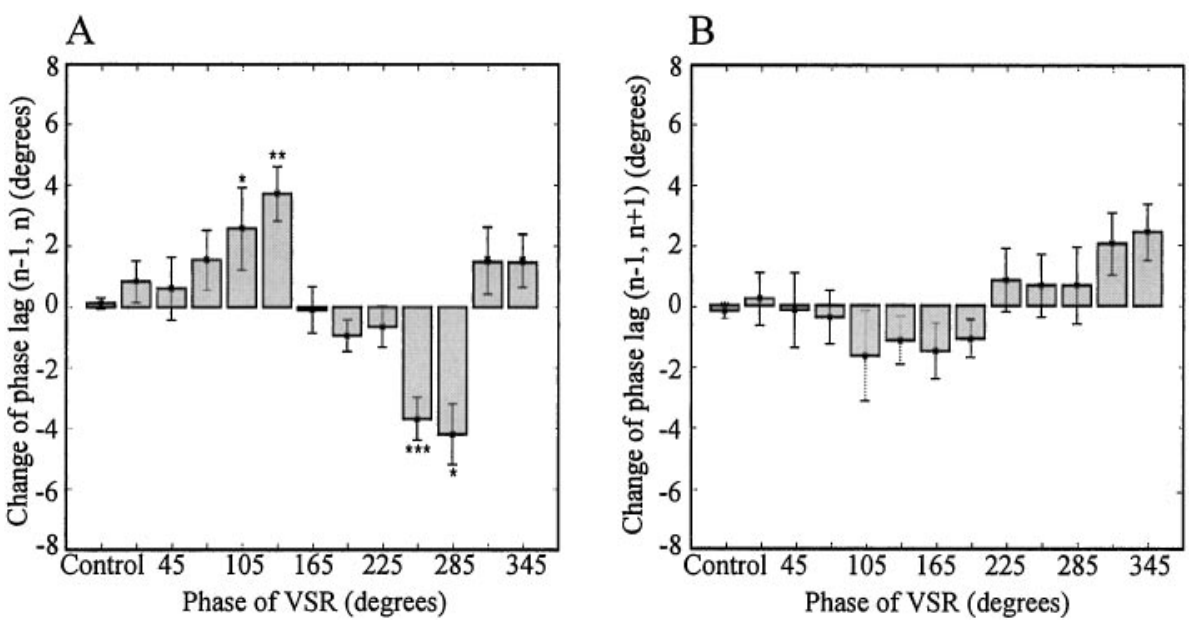

The VSRs are closely associated with the ventral longitudinal muscles and hyperpolarize when these muscles are stretched or placed under tension (Blackshaw and Thompson, 1988). The tension of these muscles is maximal at $\sim 270^{\circ}$ in the swim cycle (Kristan et al., 1974). When the muscle is actively controlled by the motoneurons, the highest tension should coincide with the shortest length. Hence, if the VSRs were length receptors, they should respond with depolarization at this phase, $270^{\circ}$. It was unexpected, therefore, that the phase of VSR oscillations in muscle-attached preparations was between 100 and $150^{\circ}$. One solution to this conundrum is that the VSRs may in fact be tension receptors, not length receptors. They thus would hyperpolarize whenever muscle tension is large (caused by either active contraction or external stretch); i.e., at a phase of $270^{\circ}$, the phase of maximal ventral contraction/tension during swimming. Depolarization in the VSRs could then be expected to be maximal at $180^{\circ}$ later in the cycle, at $\sim 90^{\circ}$, near the value we observed.

\section{Roles for the VSR-mediated changes in intersegmental phase lag}

The specific functions of the VSR-mediated modification of intersegmental phase lags remain to be elucidated. Our experiments suggest that the phase of VSRs during swimming in intact leeches is $\sim 120^{\circ}$ (Fig. $2 A$ ). Our data also show that VSR activity at this phase delays the phase of ganglion $n$, with a concomitant increase in the phase lag between ganglia $n-1$ and $n$ (Fig. $8 A$ ). This increased intersegmental phase lag is just what is needed to achieve the greater phase lags observed in intact leeches. However, VSR depolarization at $120^{\circ}$ also causes the phase lag between segments $n$ and $n+1$ to decrease (Fig. 7), leaving overall intersegmental phase lags unchanged (Fig. $8 B$ ). Thus VSR inputs to the central oscillator may be appropriate for correcting local phase relationships, perhaps in response to local perturbations.

Because of the large variation of intersegmental phase lags between adjacent ganglia, the motoneuron activity pattern generated by the nerve cord alone cannot produce efficient locomotion (Figs. 4, 5). The instantaneous phase lag during a 10-cycle swimming episode can vary more than $10^{\circ}$ (Fig. $4 A$ ). The mean phase lag of each swimming episode can differ by as much as $30^{\circ}$ among different episodes and preparations (Fig. $5 A$ ). The swimming leech expresses a smooth traveling wave, suggesting a low variability in intersegmental phase lags. The bidirectional modification of intersegmental phase relationships by the VSRs may aid in the expression of this smoothly undulating profile by the swimming leech.

It remains to be seen whether the combined actions of all VSRs in a given segment or those in multiple segments are responsible for the larger intersegmental phase lags of the intact leech. We are confident that VSRs are tension transducers that can relay information of body shape through their mechanical interactions with longitudinal muscles. The VSR properties described here together with their interactions with the swim oscillator circuits (Cang et al.,
1999) may be the means by which the intact leech adjusts local intersegmental phase relationships to accommodate environmental and developmental conditions.

\section{Sensory modification in other systems}

Three general principles for sensory modification of motor circuits are described by Pearson and Ramirez (1997). (1) Sensory feedback contributes to the generation and maintenance of rhythmic activity. (2) Phasic sensory signals initiate major phase transitions in intact motor systems. (3) Sensory signals regulate the amplitude of ongoing motor activity. In addition, for segmented animals, the sensory input must be able to modify the intersegmental phase relationships. In the absence of sensory input in the lamprey, for example, the intersegmental phase lag of the spinal cord varies greatly from cycle to cycle (Matsushima and Grillner, 1992) and for different individual preparations (Sigvardt and Williams, 1996). Using pharmacological microstimulation of the brain stem to elicit fictive swimming in longer spinal cord preparation of larval lamprey, Hagevik and McClellan (1994) found that the intersegmental phase lag is smaller than that observed in whole animals. The lamprey edge cells, stretch receptors located along the lateral margin of the spinal cord, signal the ongoing movement (Grillner et al., 1982, 1984) and have synaptic connections with locomotor neurons (Viana di Prisco et al., 1990) and thus have the potential to increase and/or stabilize the phase lags generated by the spinal cord. In the crayfish swimmeret system, two nonspiking stretch receptors (NSSRs) were found in each swimmeret-bearing segment; these depolarize in response to retraction of the swimmeret (Heitler, 1982). Sinusoidal current injected into a single NSSR produces a beat frequency modulation of spontaneously generated rhythm (Heitler, 1986). As another example of sensory modification, the locust flight rhythm can be entrained by the imposed rhythmic movement of one forewing, which is most likely mediated by the bursts of stretch receptor activity (Wendler, 1983). All of these studies suggested that the sensory input can and must contribute to intersegmental coordination. Our study thus provides explicit information about this aspect of sensory modification. It remains to be seen whether the proprioceptors in those other systems can, like the VSR, modulate intersegmental phase relationships.

\section{VSRs function as analog-digital converters}

Large and broad spikes can be elicited in the soma of VSRs (Blackshaw and Thompson, 1988), but they cannot propagate to the CNS because the axon does not sustain action potentials. In our recordings from the VSR axons, brief spontaneous depolarizations are characteristically seen. These must originate from the central terminals of the VSR because they are present even when the dendrite and soma of the VSR are removed (Fig. 1A). Blackshaw and Thompson (1988) suggested that these small depolarizations might be postsynaptic potentials, because they were abolished by 
bathing the preparation in $15 \mathrm{~mm} \mathrm{Mg}^{2+}$ saline, which blocks chemical synapses in the leech. However, the extreme sensitivity of the event frequency to small currents (Fig. $1 B$ ) makes this interpretation unlikely. Moreover, a high concentration of $\mathrm{Mg}^{2+}$ raises the impulse threshold in leeches (Pearce and Friesen, 1985). Therefore, the small spike-like depolarizations are more likely to be impulses, which disappear with elevated impulse threshold. If the small spikes are indeed action potentials initiated at the central terminal, the VSRs function as analog-digital converters. In other words, the analog signal of muscle contraction is conveyed by graded potential changes to central terminals of the VSRs, where it modulates action potential frequency.

\section{Conclusion}

We have shown that stretch receptors in the leech body wall are excellent candidates to convey information concerning the execution of the swim motor program to the central oscillator circuits. Activity in these receptors can modify local intersegmental phase lags within the nerve cord and thereby, in combination with the central oscillator, may set intersegmental phase relationships. We now view the complete leech swim oscillator in the intact animal as central oscillatory circuits intimately linked with peripheral motorsensory loops.

\section{REFERENCES}

Bevington PR (1969) Data reduction and error analysis for the physical sciences. New York: McGraw-Hill.

Blackshaw SE (1993) Stretch receptors and body wall muscle in leeches. Comp Biochem Physiol A Physiol 105:643-652.

Blackshaw SE, Kristan Jr WB (1990) Input from single stretch receptor neurons influences the centrally generated swim motor pattern in the leech. J Physiol (Lond) 425:93P.

Blackshaw SE, Thompson SWN (1988) Hyperpolarizing responses to stretch in neurons innervating leech body wall muscles. J Physiol (Lond) 396:121-138.

Brodfuehrer PD, Debski EA, O'Gara BA, Friesen WO (1995) Neuronal control of swimming. J Neurobiol 27:403-418.

Cang J, Yu X, Friesen WO (1999) Synaptic interactions between ventral stretch receptors and swim-related neurons in the medicinal leech. Soc Neurosci Abstr 25:1644.

Cohen AH, Wallen P (1980) The neuronal correlate of locomotion in fish: "fictive swimming" induced in an in vitro preparation of the lamprey spinal cord. Exp Brain Res 41:11-18.

Delcomyn F (1980) Neural basis of rhythmic behavior in animals. Science 210:492-498.

Fisher NI (1995) Statistical analysis of circular data. Australia: Cambridge UP.

Friesen WO (1981) Physiology of water motion detection in the medicinal leech. J Exp Biol 92:255-275.
Friesen WO (1989) Neuronal control of leech swimming movements. In: Cellular and neuronal oscillators (Jacklet JW, ed), pp 269-316. New York: Marcel Dekker.

Gray J (1958) The movement of the spermatozoa of the bull. J Exp Biol 35:96-108.

Grillner S, McClellan A, Sigvardt K (1982) Mechanosensitive neurones in the spinal cord of lamprey. Brain Res 235:169-173.

Grillner S, Williams T, Lagerback PA (1984) The edge cell, a possible intraspinal mechanoreceptor. Science 223:500-503.

Hagevik A, McClellan AD (1994) Coupling of spinal locomotor networks in larval lamprey revealed by receptor blockers for inhibitory amino acids: neurophysiology and computer modeling. J Neurophysiol 72:1810-1829.

Heitler WJ (1982) Non-spiking stretch-receptors in the crayfish swimmeret system. J Exp Biol 96:355-366.

Heitler WJ (1986) Aspects of sensory integration in the crayfish swimmeret system. J Exp Biol 120:387-402.

Kristan WB, Calabrese RL (1976) Rhythmic swimming activity in neurons of the isolated nerve cord of the leech. J Exp Biol 65:643-668.

Kristan WB, Stent GS, Ort CA (1974) Neuronal control of swimming in the medicinal leech. I. Dynamics of the swimming rhythm. J Comp Physiol 94:97-120.

Marder E, Calabrese RL (1996) Principles of rhythmic motor pattern generation. Physiol Rev 76:687-717.

Matsushima T, Grillner S (1992) Neural mechanisms of intersegmental coordination in lamprey: local excitability changes modify the phase coupling along the spinal cord. J Neurophysiol 67:373-388.

Murchison D, Chrachri A, Mulloney B (1993) A separate local patterngenerating circuit controls the movement of each swimmeret in crayfish. J Neurophysiol 70:2620-2631.

Pearce RA, Friesen WO (1984) Intersegmental coordination of leech swimming: comparison of in situ and isolated nerve cord activity with body wall movement. Brain Res 299:363-366.

Pearce RA, Friesen WO (1985) Intersegmental coordination of the leech swimming rhythm. II. Comparison of long and short chains of ganglia. J Neurophysiol 54:1460-1472.

Pearson KG (1993) Common principles of motor control in vertebrates and invertebrates. Annu Rev Neurosci 16:265-297.

Pearson KG, Ramirez J (1997) Sensory modulation of pattern-generating circuits. In: Neurons, networks, and motor behavior (Stein PSG, Grillner S, Selverston AI, Stuart DG, eds), pp 225-235. Cambridge, MA: MIT.

Sigvardt KA, Williams TL (1996) Effects of local oscillator frequency on intersegmental coordination in the lamprey locomotor CPG: theory and experiment. J Neurophysiol 76:4094-4103.

Viana di Prisco G, Wallen P, Grillner S (1990) Synaptic effects of intraspinal stretch receptor neurons mediating movement-related feedback during locomotion. Brain Res 530:161-166.

Weeks JC (1981) Neuronal basis of leech swimming: separation of swim initiation, pattern generation, and intersegmental coordination by selective lesion. J Neurophysiol 45:698-723.

Wendler G (1983) The interaction of peripheral and central components in insect locomotion. In: Neuroethology and behavioral physiology (Huber F, Markl H, eds), pp 42-53. Berlin: Springer.

Yu X, Nguyen B, Friesen WO (1999) Sensory feedback can coordinate the swimming activity of the leech. J Neurosci 19:4634-4643. 